TENDENCIAS

Revista de la Facultad de Ciencias

Económicas y Administrativas.

Universidad de Nariño

ISSN-E 2539-0554

Vol. XXI No. 2 - 2do Semestre 2020,

Julio-Diciembre - Páginas 106-123

\title{
EL NIVEL EDUCATIVO COMO EJE FUNDAMENTAL PARA LA INTERNACIONALIZACIÓN Y DESARROLLO EMPRESARIAL ${ }^{1}$
}

\author{
THE EDUCATIONAL LEVEL AS A FUNDAMENTAL AXIS FOR \\ INTERNATIONALIZATION AND BUSINESS DEVELOPMENT
}
O NÍVEL EDUCACIONAL COMO EIXO FUNDAMENTAL PARA A INTERNACIONALIZAÇÃO E O DESENVOLVIMENTO DE NEGÓCIOS

FERNÁNDEZ HURTADO_Saúl Rick; MARTÍNEZ MARTÍNEZ_Luz Ángela; NGONO FOUDA_ Regine Adele

\begin{abstract}
Post-doctorate in Globalization and Regional Development, Shanghai University, China. Profesor e Investigador Institución Universitaria Escuela Nacional del Deporte, Programa de Mercadeo y Negocios Internacionales. E-mail: srickfernandez@endeporte.edu.co, Colombia.

Profesional en Ingeniería Industrial, Departamento de Operaciones y Sistemas, Universidad Autónoma de Occidente. E-mail: luz_angela.martinez@uao.edu.co, Colombia.

Ph.D., in Logistic Management and Engineering, Shanghai Maritime University, China. Master in international trade, East China University of Science \& Technology, China. E-mail: reginemilena@yahoo.fr, China.
\end{abstract}

Recibido: 3 de febrero de 2020

Aprobado: 22 de junio de 2020

DOI: https://doi.org/10.22267/rtend.202102.143

\section{RESUMEN}

El objetivo principal de esta investigación es identificar la influencia que ejerce el nivel formativo de los empresarios en el desarrollo de las negociaciones a nivel internacional; el enfoque que se utilizó fue a través del análisis descriptivo y empírico; bajo metodología exploratoria, respecto a

\footnotetext{
${ }^{1}$ Este artículo es producto del Proyecto de Investigación Titulado: Cluster empresarial en el Valle del Cauca como mecanismo a la internacionalización de mercados. Proyecto vinculado al grupo de investigación GICEAD de la Facultad Ciencias Económicas y de la Administración, Institución Universitaria Escuela Nacional del Deporte.
} 
las variables medidas, lo cual ayudó al entendimiento de lo que requieren las empresas a través de las habilidades de los egresados universitarios.

Como resultado de esta investigación se obtuvo una necesidad a nivel empresarial y gubernamental, evidenciando que la formación universitaria deberá tener un cambio ante el saberhacer (macro), lo cual es una necesidad para el éxito empresarial a nivel internacional. La investigación permite concluir aspectos relevantes ante actividades empresariales, donde la correlación de fortalecimiento empresa-gobierno-universidad, está produciendo un efecto negativo, y mediante la obtención psicoempresarial, bajo el análisis de resultados obtenidos mediante los datos recolectados en la investigación.

Palabras clave: globalización; mercados internacionales; sistema formativo; organizaciones; estructura educativa; psicoempresariales.

JEL: F15; H32; I25; I28; M16

\begin{abstract}
The main objective of this research is to identify the influence of the training level of entrepreneurs in the development of international negotiations. The approach used was through descriptive and empirical analysis, with respect to measured variables, which helps the understanding of what companies require through the skills of university graduates.
\end{abstract}

As a result of this article, a need was obtained at the corporate and governmental level, that university training should have a change to the know-how (macro), which is a necessity for international business success. The research allows to conclude aspects relevant to business activities, where the correlation of strengthening company-government-university, is producing a negative effect, and through the psycho-entrepreneurial acquisition.

Keywords: globalization; international markets; formative system; organizations; educational structure; psychoentrepreneurs.

JEL: F15; H32; I25; I28; M16 


\section{RESUMO}

O principal objetivo desta pesquisa é identificar a influência exercida pelo nível de formação de empreendedores no desenvolvimento de negociações em nível internacional; A abordagem utilizada foi a análise descritiva e empírica, referente às variáveis mensuradas, o que auxilia na compreensão do que as empresas exigem através das habilidades dos graduados da universidade.

Como resultado desta pesquisa, obteve-se uma necessidade nos níveis empresarial e governamental, que da mesma forma que a educação universitária deve ter uma mudança em face do know-how (macro), que é uma necessidade de sucesso nos negócios em nível internacional. A investigação permite concluir aspectos relevantes em face das atividades empresariais, onde a correlação do fortalecimento empresa-governo-universidade está produzindo um efeito negativo e, através da obtenção de psicoempresas, sob a análise dos resultados obtidos através dos dados coletados na pesquisa.

Palavras-chave: globalização; mercados internacionais; sistema formativo; organizações; estrutura educacional; psicoempresários

JEL: F15; H32; I25; I28; M16

\section{INTRODUCCIÓN}

El presente artículo de investigación, explora las variables requeridas ante el desarrollo empresagobierno-universidad, donde la integración del conocimiento de los empresarios es pertinente, e igual el fortalecimiento empresarial para la toma decisiones es necesario en la medida de obtener recursos.

La investigación incorpora, además, otras características interesantes que ayudan a determinar el desarrollo del problema planteado como los incentivos a empresarios, la falta de personal cualificado, regulaciones gubernamentales, carencia de conocimiento, información cultural y la necesidad de recursos tecnológicos, los cuales son las variables de análisis de investigación.

La metodología empleada en el artículo de investigación se realizó con la finalidad de analizar y conocer el entorno psicoempresairal como un problema principal (Robayo, 2016) y el comportamiento de los empresarios ante el nivel de educación respecto a las habilidades del “saber" entregadas por las universidades, por lo tanto la medición empírica fue relevante. 
El objetivo del artículo de investigación es determinar las causas que hicieron parte del estudio las cuales fueron relevantes y que ocasionan el desconocimiento cultural en el ámbito empresarial y económico, como también realizar un análisis descriptivo y empírico bajo la investigación a nivel formativo-empresarial, obteniendo un resultado bajo los datos de investigación.

Finalizando, el presente artículo de investigación se estructura de la siguiente forma: en la primera sección se identifica el conocimiento sobre el problema planteado, seguido del desarrollo teórico; por lo tanto se resaltan diversos autores que amplían y aclaran el aporte al conocimiento por parte de los escritores del presente estudio, finalmente se presenta el análisis descriptivo y empírico que ayuda a determinar el entorno de las variables que se estudiaron, exponiendo así un breve análisis y unas conclusiones con base a los resultados conseguidos.

\section{REFERENTES TEÓRICOS}

\section{Sistema formativo hacia los empresarios}

La globalización o apertura comercial es un mecanismo fundamental para el desarrollo y transformación de la economía de un país, por ende, la formación idónea de los integrantes que participan de manera activa en el comercio internacional; es de suma importancia para el avance económico de las organizaciones, sectores, países etc.

De ahí el papel trascendental que deben cumplir las instituciones educativas y el Estado (Hernández, Martucelli, Moctezuma, Muñoz y Narro, 2015); para garantizar los instrumentos adecuados en la capacitación de los empresarios, sobre todo a aquellos que lideran el proceso gerencial de las mipymes, los cuales poseen poco conocimiento y experiencia en el tema de establecimiento de relaciones internacionales exitosas, y así de esta manera poder contribuir a la superación de las distintas falencias que se han observado en el transcurso de los acuerdos comerciales (Fernández, 2014a; Botello, 2016).

Una de las problemáticas que más preocupa es el desconocimiento y formación en el ámbito cultural, social, y todos aquellos aspectos esenciales de la interculturalidad que deben tener en cuenta las organizaciones a la hora de implementar cada una de sus estrategias, las cuales les permitirá penetrar otros mercados, diversificar y expandir sus productos y servicios. 
Pues solo se daba por sentado el "éxito" de los acuerdos en la disponibilidad de tener ventajas en los recursos naturales; o de poseer una posición geográfica estratégica, pero este aspecto que están relevante pasa por desapercibido por las instituciones universitarias, debido a que el enfoque se centra sobre los recursos monetarios e incentivos para solventar todos los gastos que conlleva al incursionar en otros países.

Hipótesis 1a. El aumento de la capacidad de aprendizaje cultural de otros mercados, refuerza la relación de empresarios a nivel internacional en cuanto al número de transacciones empresariales.

Como se puede observar desde hace mucho tiempo el sistema educativo en general presenta falencias que a la luz de hoy han sido mínimos o pocos, los esfuerzos por parte de las entidades competentes por formar profesionales idóneos en las diferentes áreas importantes, las cuales le permitirán a Colombia a través de sus empresarios y empresas alcanzar la competitividad a nivel internacional (Fernández y Martinez, 2018; Vanegas, Restrepo y González, 2015), temas como la ciencia y la tecnología son primordiales para alcanzar dicha competitividad, así como otros factores fundamentales como la investigación.

El sistema educativo actual no cumple con las exigencias y estándares internacionales en materia de acreditaciones en las diferentes áreas relevantes para la competitividad internacional (Hernández et al. 2015), en Colombia no existe una institución en la educación superior enfocada de lleno al énfasis del desarrollo y fomento empresarial, que abarque de manera congruente y adecuada las competencias interculturales de los empresarios, el cual sin duda sería de gran beneficio al crecimiento económico del país, en el cual primero se debe garantizar la calidad de los profesores que pertenecen a la educación superior.

Colombia no ha sabido aprovechar estos diferentes canales de información para atenuar de manera significativa las deficiencias que existen en el entorno académico, una de las propuestas sería establecer o reafirmar los lazos ya existentes, o buscar opciones y alternativas las cuales ayuden a subsanar tales deficiencias.

Hipótesis 1b. El aumento de la capacidad de aprendizaje cultural de otros mercados por parte de las empresas, minimiza el apoyo gubernamental reflejado ante el número de transacciones empresariales. 


\section{Escenarios de formación a empresas para el mercado}

Actualmente lo más difícil para emprender en un mercado es la falta de conocimiento de proyectos en los cuales el gobierno no ha realizado la gestión de darlos a conocer; lo más idóneo para que los empresarios que tienen iniciativa de emprender es poder contar con información clara y contundente de sus competidores, las empresas son parte de nuestro entorno (Bucardo, Saavedra y Camarena, 2015; Fernández, 2016), aunque todas son diferentes y para poder emprender es necesario un estudio donde abarque mayores dimensiones para poder entrar a un mercado que día a día se globaliza.

La globalización ha sido rápida durante las últimas décadas, uno de los mayores afluentes ha sido la revolución tecnológica de la información y las telecomunicaciones que se experimentan a nivel mundial (Martinez y Fernández, 2018). El rápido crecimiento de mercado hace que los empresarios tengan mayores niveles de capacitación, para poder competir y ganar posicionamiento en un mercado.

Hipótesis 2a. Toda la falta de recursos constantes, afectará negativamente la rentabilidad empresarial.

De acuerdo a Botero (2013) "El entorno de la apertura económica y de las compañías constituyen un escenario en el cual las fases de gestión empresarial deben ser examinadas de manera no habitual, a la luz de los retos que se desprenden de este ambiente".

El gobierno se preocupa por lograr una mayor economía donde se generé empleo y productividad al país, Colombia cuenta con una economía sólida pero con muchas dificultades psicoempresariales (Contreras y Rozo, 2015), por ende, se intentó reducir el proteccionismo, integración al mundo (Fernández, 2014b; Navarrete, 2017) abrir mercados y competir saliendo a la búsqueda de una Colombia moderna, gracias a eso hoy en día se enfrentan a un mundo de grandes y pequeñas barreras que se necesitan superar con una determinación, (Armario, Rastrollo y González, 2009; Momberg, 2016) si la misma determinación con la que empezamos se hubiera mantenido hoy se contaría con un país más depurado en sus procesos, más ágil en sus procedimientos y en los tramites, con menos exigencias y mejores prácticas. 
Hipótesis 2 b. Toda la cooperación constante a nivel gubernamental, afectará positivamente la rentabilidad empresarial.

El gobierno colombiano ha realizado muchas alianzas donde se permite la libre circulación de bienes y servicios, pero para los empresarios que no conocen las oportunidades fuera de las fronteras (Ngono, Darcis, Muhammad y Fernández, 2014), esto significa tener barreras psicoempresariales (Robayo, 2016), esto sucede por el desconocimiento de los proyectos del gobierno, son pocas las empresas que adquieren estos proyectos de educación y ayudas monetarias, es por eso que se pierden tantas oportunidades de mercados nuevos y competitivos por que las iniciativas están; pero las pymes desafortunadamente no cuentan con la suficiente información que les permita hacer parte de los proyectos gubernamentales (García, Gálvez y Maldonado, 2016; Fernández, 2013), la actividad innovadora sobre el crecimiento y el rendimiento de las Mipymes de los países conformados por la alianza del pacifico ha obtenido un resultado de innovación de su producción, en procesos y gestión que impacta positivamente el crecimiento, el empleo, las ventas y así mismo su utilidad.

Es cierto que Colombia tiene muchos acuerdos comerciales donde se podría realizar un aporte económico generando más empleo, y más oportunidades de mercado que aún están perdidas, es por eso que es necesario conocer nuestros acuerdos con los demás países, y capacitarnos para alcanzar los objetivos que cada empresa emprendedora desea, por lo tanto, no es necesario establecer más tratados de libre comercio en un periodo de por lo menos 8 años, y enfocarse a estimular las empresas y los mercados ya firmados bajo el TLC.

\section{Innovación empresarial en pro de ser competitivos}

Como ha hecho referencia Larrea (2009), para poder cumplir con dicho avance económico, las organizaciones deben identificar un sin número de características y requerimientos para llegar a ser competentes a nivel local y, aún más en el ámbito internacional, entre estas características se puede destacar como eje principal la innovación.

Pabón (2016) indica que "La innovación desde la perspectiva de las sociedades comerciales y las organizaciones empresariales es la comercialización exitosa de una invención, entrando en un mercado o creando uno nuevo, o la mejora de procesos internos y la generación de eficiencias". 
Este es un punto diferenciador entre una empresa y otra en un mercado que se encuentra globalizado y saturado de productos, servicios y procesos innovadores; es necesario tener en cuenta que la innovación va más allá de la incorporación de tecnología empresas (Fernández, Diaz, Rodríguez y Martinez, 2019; Cano y Baena, 2015), sino que, además, está se centra en prever las necesidades del mundo globalizado, donde los consumidores son más exigentes, y por consiguiente este escenario permitirá perdurar en el mercado nacional e internacional.

Aguado (2005) mencionó “Los incrementos de productividad inducidos por la innovación no sólo hacen que las empresas de un territorio sean competitivas, sino que contribuyen a elevar el nivel de vida de los ciudadanos de ese territorio".

La productividad es un factor clave y va encaminada sin duda alguna de la innovación, por eso las empresas y los gobiernos deben orientar sus estrategias, políticas y recursos a mejorar gran parte las falencias que se presentan a nivel psicoempresarial; que además de generar productividad, genera que las organizaciones lleguen a ser competitivas.

En relación con lo anterior se requiere que la inversión en desarrollo científico, tecnológico e investigativo en las empresas sea significativa y se genere una colaboración de los diferentes estamentos gubernamentales, los cuales brinden las herramientas adecuadas para que puedan competir de manera efectiva, debido a que para conseguir la innovación se necesita de la infraestructura y el acceso a los recursos (capital y humano) idóneos.

No obstante, también se necesitan políticas coherentes que estén acordes a todos los procesos innovadores, al igual que un marco normativo que propenda por el mismo, nuevos enfoques estratégicos de las nuevas empresas; personal calificado y cualificado, la integración empresauniversidad-sector privado-sector público (Fernández, Castillo y Martínez, 2018), estos últimos deben actuar de forma sincronizada, pues cada uno de ellos se alimenta de los demás.

Se puede observar que desde hace mucho tiempo el tema de la innovación se volvió en un foco de vital importancia para los países más desarrollados, lo que les ha permitido llevar a la vanguardia dicho tema. 
Lamentablemente este proceso de industrialización para Colombia se ha desarrollado de manera paulatina; lo que no le permite ir al ritmo de la evolución económica global. El contexto internacional muestra que los niveles más altos de desarrollo lo poseen aquellos países que gozan de altos índices de emprendimiento-innovador en sus economías. Colombia debe identificar y hacer un diagnóstico exhaustivo a nivel empresarial sobre los puntos críticos en los que es necesario actuar y corregir (Benavides, 2015).

La postura que toman algunos empresarios en relación con las negociaciones internacionales que realizan, de cierta forma influyen, no solo económicamente si no que afecta a los pequeños empresarios y emprendedores que están abriendo camino en este ámbito empresarial (Botello, 2016), y es debido a esto que se hace hincapié en la formación idónea del talento humano.

\section{METODOLOGÍA}

En esta investigación se utilizó la metodología exploratoria con análisis descriptivo como también empírica, y se realizó por medio de un estudio de tipo hipotética-deductiva la cual ayudó a definir el estado actual de las deficiencias del nivel formativo, ya que no es lo que los empresarios esperan por parte de las entidades, sino, la labor de identificar las falencias que afectan las negociaciones actualmente.

\section{Muestra poblacional}

La muestra poblacional estuvo constituida por una población de 145 bajo un nivel de confianza del $95 \%$ y margen de error del $5 \%$, respecto a lo anterior se estableció un estudio con una muestra de 105 empresarios y profesionales en diferentes carreras, para lo cual se realizaron encuestas con 5 preguntas, donde se interrogó sobre cómo se considera el estado actual a nivel formativo para el desarrollo de empresarios y qué aportes se conocen por parte de los empresarios que beneficie a la sociedad, el cálculo fue realizado bajo el método estadístico para determinar la muestra de población finita (Formula 1), mediante la muestra, se realizó la tabulación necesaria para poder obtener datos reales, medibles y alcanzables, teniendo como resultado análisis de correlación.

$\alpha$ : Nivel de confianza

$$
\frac{N *\left(\alpha_{c} * 0,5\right)^{2}}{1+\left(e^{2} *(N-1)\right)}=
$$

e: Margen de error

N: Tamaño Población 


\section{RESULTADOS Y DISCUSIÓN}

La Tabla 1 se detalla la correlación de siete variables de los entornos empresariales utilizados estadísticamente. Las correlaciones entre las variables fueron positivas, por lo tanto existe una relación estadísticamente significativa entre las variables de la muestra.

\section{Tabla 1}

Estadística correlacional

\begin{tabular}{|c|c|c|c|c|c|c|c|c|c|}
\hline Variables & Promedio & $\begin{array}{l}\text { Deviación } \\
\text { Estándar }\end{array}$ & 1 & 2 & 3 & 4 & 5 & 6 & 7 \\
\hline $\begin{array}{l}\text { 1. Incentivo a } \\
\text { empresarios }\end{array}$ & 0,32 & 0,47 & 1 & .35 & $-.19^{\mathrm{a}}$ & -.48 & -.37 & .30 & $-.20^{\mathrm{a}}$ \\
\hline $\begin{array}{l}\text { 2. Falta de personal } \\
\text { cualificado }\end{array}$ & 0,56 & 0,50 & & 1 & $-.11^{\mathrm{a}}$ & -.08 & -.14 & -.01 & .11 \\
\hline $\begin{array}{l}\text { 3. Regulaciones } \\
\text { gubernamentales }\end{array}$ & 0,35 & 0,48 & & & 1 & $0,19^{-}$ & $\begin{array}{c}- \\
.10^{\mathrm{a}}\end{array}$ & .29 & -.37 \\
\hline $\begin{array}{l}\text { 4. Ayudas } \\
\text { gubernamentales }\end{array}$ & 0,41 & 0,49 & & & & 1 & $.66^{\mathrm{b}}$ & -.65 & $.44^{\mathrm{b}}$ \\
\hline $\begin{array}{l}\text { 5. Carencia de } \\
\text { conocimientos }\end{array}$ & 0,23 & 0,42 & & & & & 1 & $-.43^{\mathrm{a}}$ & .29 \\
\hline $\begin{array}{l}\text { 6. Interés por } \\
\text { información cultural }\end{array}$ & 0,38 & 0,49 & & & & & & 1 & $-.68^{b}$ \\
\hline $\begin{array}{l}\text { 7. Falta de recursos } \\
\text { tecnológicos }\end{array}$ & 0,78 & 0,41 & & & & & & & 1 \\
\hline
\end{tabular}

${ }^{a}$ Nivel de significancia $\mathrm{P}<.05$.

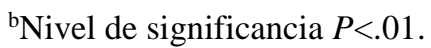

Fuente: elaboración por los autores (2019).

Basados en la muestra, se puede observar que se cuenta con una insatisfacción por la carencia de la educación brindada por el estado colombiano, donde no se proporciona la educación idónea para ser competitivos y generar estrategias de crecimiento económico; sin embargo, se observa que el $\mathrm{R}^{2}$ indica que el modelo ajustado explica $40,6 \%$ de la variabilidad en el indicador falta de personal cualificado frente a las regulaciones emitidas a nivel gubernamental. El coeficiente de correlación es igual a 0,64, indicando una relación moderadamente fuerte entre las variables. El error estándar estimado indica que la desviación estándar de los residuos es 0,4; la posición de respuesta es negativa; aun así, se contempla de que la mayoría sienten un apoyo por parte del gobierno e instituciones educativas, pero si cuentan con la necesidad de incursionar en un mercado, ya que 
hoy en día, quien no goce con la capacidad de proyectarse como empresario, lastimosamente su nivel de vida no mejorará.

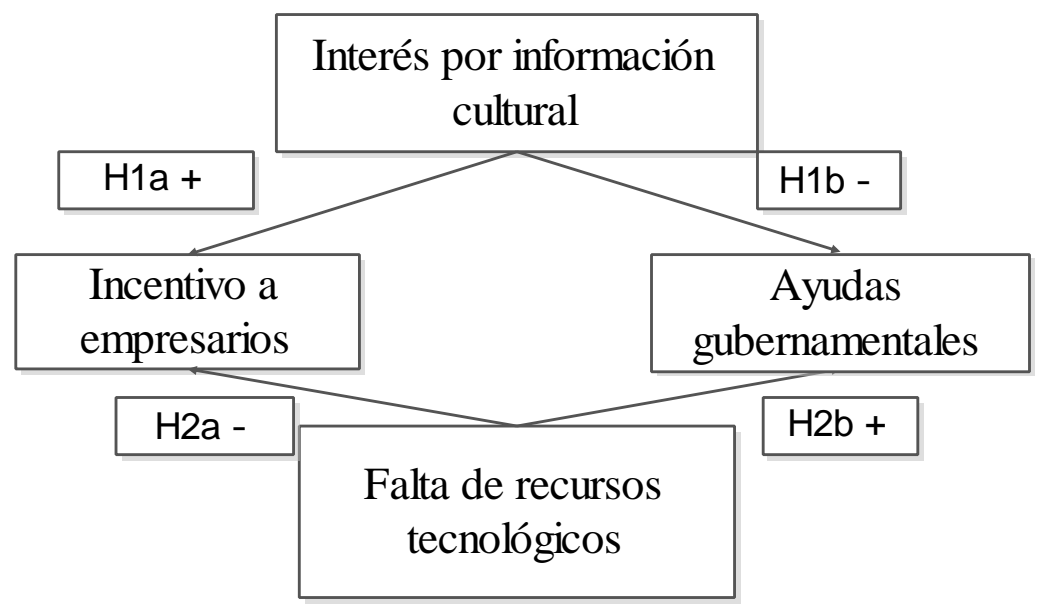

Figura 1. Modelos de investigación.

Fuente: elaboración por los autores (2019).

Al momento de analizar los resultados de la muestra, se concluye que hoy en día las personas con aspiración a ser empresarios tienen gran desconocimiento en aspectos culturales, se puede observar no se informa de la interculturalidad por la que atraviesa el mundo dado que se requiere mayor nivel de recursos tecnológicos, así se ve en la Tabla 1 en donde la correlación es negativa entre estas variables (-.68), presentando un valor $P$ de 0,000 , (ver Tabla 2) lo cual indica el análisis de varianza que el valor $P$ que tiene una relación estadísticamente significativa entre las variables de la medida, aun así tiene un nivel de confianza de $95 \%$.

\section{Tabla 2}

Regresión Simple - Falta de recursos tecnológicos vs. Ayudas Gubernamentales

\begin{tabular}{lrrrrr}
\hline \multicolumn{1}{c}{ Fuente } & Suma de Cuadrados & Gl & Cuadrado Medio & Razón-F & \multicolumn{1}{c}{ Valor-P } \\
\hline Modelo & 22,5778 & 1 & 22,5778 & 1145,13 & 0,0000 \\
Residuo & 1,9322 & 98 & 0,0197164 & & \\
Total (Corr.) & 24,51 & 99 & & & \\
\hline
\end{tabular}

Fuente: elaboración por los autores (2019).

Por otro lado, la medida estadística $R^{2}$ indica que el modelo ajustado explica 92,12\% la variable de falta de recursos tecnológicos, por ello, el coeficiente de correlación (-.960) indica una relación relativamente fuerte entre ambas variables, por tanto la hipótesis $(\mathrm{H} 2 \mathrm{~b}+)$ es positiva, sin dudarlo esto se debe a la globalización que nos permite un intercambio no sólo de bienes y servicios 
(Suárez y Muñoz, 2016), sino también de apoyo gubernamental y recursos tecnológicos necesarios para el desarrollo empresarial; en vez de ver esto como una desventaja, debería ser un abre bocas para todos aquellos que están dispuestos a ser grandes empresarios (Santos y García, 2016), ya que Colombia es un país con gran biodiversidad y posibilidades infinitas para aprovechar esta cualidad para darse a conocer internacionalmente. De acuerdo a la muestra se considera que se carecen de conocimientos tanto, para llevar a cabo una actividad empresarial y de igual forma obtener los recursos para llegar a incrementar prospectos económicos (Flores y Vanoni, 2016), por tanto, se carece de incentivos mediante las entidades avaladas para tener apoyo que no signifique mayores costos empresariales; por otro lado, se tiene consciencia de que no se cuenta con tecnología apta para competir con grandes multinacionales, y por este las pymes requieren de apoyo. Es claro de que el nivel económico en Colombia es aprovechado por compañías fuertes económica y tecnológicamente, lo cual complica competir con este tipo de compañías, ya que poseen un amplio reconocimiento; financiamiento, con personal calificado y alta capacidad innovadora.

La mayoría de los encuestados, mostró preocupación respecto a estas variables, debido a que consideran que se encuentran en un país con oportunidades de crecimiento, ya que creen que cada día es más complejo solicitar un empleo así como se muestra en la correlación entre las variables, de este modo se observa que la correlación dio negativo (-.48), y dado que el Valor- $P$ es mayor a 0,05, lo que confirma que no existe relación estadísticamente significativa entre las variables (ver Tabla 3), y mediante un nivel de confianza del 95\%, ya que después de muchos años y esfuerzos académicos e inversiones, no son favorablemente retribuidos financieramente en el entorno laboral como se debería; las capacidades son medidas para obtener un salario adecuado, y las exigencias no son aptas para un profesional que apenas está terminando su carrera universitaria, dando lugar a una escasez de talento y generando un mayor índice de desempleo.

\section{Tabla 3}

Regresión Simple - Falta de recursos tecnológicos vs. Incentivos a Empresarios

\begin{tabular}{lrrrrr}
\hline \multicolumn{1}{c}{ Fuente } & Suma de Cuadrados & Gl & Cuadrado Medio & Razón-F & \multicolumn{1}{c}{ Valor-P } \\
\hline Modelo & 0,19125 & 1 & 0,19125 & 1,10 & 0,2959 \\
Residuo & 16,9688 & 98 & 0,173151 & & \\
Total (Corr.) & 17,16 & 99 & & & \\
& & & & &
\end{tabular}

Fuente: elaboración por los autores (2019). 
El estadístico $R$-Cuadrada indica que el modelo ajustado explica $1,114 \%$ de la variabilidad en falta de recursos tecnológicos frente al incentivo a empresarios. Por ende, el coeficiente de correlación es igual a 0,1055, indicando una relación relativamente débil entre las variables, por tanto, la hipótesis (H2a-) indica que es negativa. E incluso la desviación estándar es 0,416 lo cual expresa una aproximación al valor 0 , y esto menciona que no hay correlación estadística entre las variables dado que el valor $P$ es mayor o igual a 0,05 .

Es necesario encontrar una estrategia que permita disminuir el desempleo y aumentar el personal calificativo, es de vital importancia realizar una evaluación al sistema educativo (Moreno, 2015; San Martín, Jiménez y Sánchez, 2016), así encontrar las falencias y poder desarrollar y estudiar soluciones de cambio; por ello, es necesario realizar convenios con compañías que exijan la estimulación de personal idóneo, así mismo se comprende por el análisis de $R^{2}$, lo que indica que el modelo explica la variabilidad entre los indicadores de medición (como se observa en la Tabla 3) mediante el $26,81 \%$ y el coeficiente de correlación 0,52 , lo que indica una relación moderadamente fuerte entre las variables, entendiendo esto, es necesario tener incentivos al desarrollo empresarial.

La Tabla 4, muestra que el valor $P$ es menor que 0,05 , por consiguiente existe una relación estadísticamente significativa entre las variables, además cuenta con un nivel de confianza del 95,0\%, por tanto respecto a la hipótesis $(\mathrm{H} 1 \mathrm{a}+)$ es positiva.

\section{Tabla 4}

Regresión Simple - Intereses por información cultural vs. Incentivos a empresarios

\begin{tabular}{lrrrrr}
\hline \multicolumn{1}{c}{ Fuente } & Suma de Cuadrados & \multicolumn{1}{c}{ Gl } & Cuadrado Medio & Razón-F & \multicolumn{1}{c}{ Valor-P } \\
\hline Modelo & 1,07654 & 1 & 1,07654 & 4,69 & 0,0327 \\
Residuo & 22,4835 & 98 & 0,229423 & & \\
Total (Corr.) & 23,56 & 99 & & & \\
\hline
\end{tabular}

Fuente: elaboración por los autores (2019).

De igual forma, se tiene que el coeficiente de correlación es igual a 0,21, lo que indica una relación relativamente débil entre estas variables, lo cual explica que el $R^{2}$ es igual a $4,55 \%$ correspondiente al modelo ajustado. 
De acuerdo a los resultados arrojados, son varios los interrogantes a estudiar. Son amplios los deseos de las personas en proceso de formación, ser profesionales calificados que puedan desenvolverse en el ambiente de su interés, pero se encuentran con el amplio desconocimiento de las regulaciones gubernamentales adoptadas por el gobierno colombiano; siendo así, una gran barrera en general es el desconocimiento de las ampliaciones ante las actividades empresariales. Por supuesto, son muchas las personas con deseo de incursionar en un mercado, pero no cuentan con la información necesaria, dejando las ideas y propuestas en papel (caso universitario), ya que no conocen los procesos debidamente parametrizados para la fijación de esa idea. Eso hace parte de la formación personal, pero también con gran influencia, en la escasa participación ciudadana en la toma de decisiones nacionales y el déficit de información, ya sean acuerdos, impuestos, barreras, reformas, entre otras; haciendo al ciudadano ignorante de las cuestiones legales y regulatorias del país, dándole una gran muralla por mucho camino por recorrer, para poder bajar ese telón que el gobierno ha querido ponerle a la ciudadanía empresarial para tomar decisiones sin pensar en el bienestar de la sociedad.

En la Tabla 5, se observa que el valor $P$ es menor que 0,05 , por consiguiente, existe una relación estadísticamente significativa entre las variables, además cuenta con un nivel de confianza del $95,0 \%$.

\section{Tabla 5}

Regresión Simple - Intereses por información cultural vs. Ayudas gubernamentales

\begin{tabular}{lrrrrr}
\hline \multicolumn{1}{c}{ Fuente } & Suma de Cuadrados & Gl & Cuadrado Medio & Razón-F & \multicolumn{1}{c}{ Valor-P } \\
\hline Modelo & 2,276 & 1 & 2,276 & 10,48 & 0,0016 \\
Residuo & 21,284 & 98 & 0,217184 & & \\
Total (Corr.) & 23,56 & 99 & & & \\
\hline
\end{tabular}

Fuente: Elaboración por los autores (2019).

Por lo anterior, tenemos el $R^{2}$ que indica un modelo ajustado de $9,66 \%$ respecto a la variabilidad de estos indicadores, por tanto, el coeficiente de correlación es igual a 0,31, indicando una relación relativamente débil en relación a las variables medidas, por este motivo se tiene que la hipótesis (H1b-) es negativa. 
Por ello, la mayoría de los empresarios hoy en día prefieren obtener una deuda con una entidad financiera, porque los incentivos que el gobierno otorga son casi que imposibles, debido a que deben de realizar actividades que les representa mayor tiempo, y que al final no se sabe si los proyectos sean aptos o simplemente las entidades tienen como respuesta, no cumple con los requisitos. Gran parte de la muestra no conoce las ayudas y tampoco se interesan por buscarlas o pedir información, las ayudas de los entes gubernamentales, están destinadas para empresas que ya están en desarrollo empresarial, por este la correlación dio negativo (ver Tabla 1). En relación con lo anterior, es claro que hay una falencia en el sistema formativo hacia los empresarios, la falta de incentivar los proyectos y la poca información que otorga las entidades competentes, las excesivas regulaciones para obtener las ayudas cada vez desmotivan y retroceden la competencia a nivel global, porque estamos en un mundo globalizado que necesita que los empresarios se encuentren capacitados para los intercambios empresariales.

\section{CONCLUSIONES}

De acuerdo a los objetivos planteados, se llegó a las siguientes conclusiones:

La principal falencia del desconocimiento cultural al momento de realizar una negociación, es primordialmente por el mínimo apoyo gubernamental, con el que cuenta la educación Colombia, convirtiendo a los futuros profesionales en personal ambiguo e ignorante. Además, los entes reguladores de estos apoyos para emprendimientos, deben de tomar las iniciativas y cambiar los procesos de selección, haciendo más posible la incursión de personas que no poseen un porcentaje de recursos idóneo, pero que si cuentan con el ideal y la motivación para realizarlas.

De acuerdo a la muestra realizada, se puede llegar a analizar el desconcierto de la población en proceso de formación por la educación, y los recursos que brindan las instituciones educativas; es por ello que de acuerdo a los porcentajes arrojados, se puede deducir las inconformidades y preocupaciones que poseen por el desconocimiento de información importante para tener en cuenta en el momento de las negociaciones internacionales; es muy claro, de que el mundo está en un momento de globalización, por ello es necesario que los países se concentren en fases de evolución, visualizar metas y objetivos para impulsar las actividades comerciales de las empresas. 


\section{REFERENCIAS}

(1) Aguado, R. (2005). Las nuevas herramientas de la política de innovación: los sistemas de innovación y el desarrollo de clusters. Boletin de Estudios Economicos, 60(186), 413-429.

(2) Armario, J. M., Rastrollo, M. A. y González, E. M. (2009). La internacionalización de la empresa: el conocimiento experimental como derterminante del resultado en mercados exteriores. Cuadernos de Economia y Direccion de la Empresa, 12(39), 123-150.

(3) Benavides, G. F. (2015). Las políticas públicas del turismo receptivo colombiano. Suma de Negocios, 6(13), 66-73. doi: 10.1016/j.sumneg.2015.08.005

(4) Botello, H. A. (2016). Las certificaciones de calidad y la internacionalización de las firmas industriales colombianas. Suma de Negocios, 7(16), 73-81. doi: 10.1016/j.sumneg.2016.02.009

(5) Botero, L. D. (2013). La globalización de la economía y la internacionalización de la empresa: una mirada en el tiempo. Revista Ciencias Estratégicas, 22(30), 203-208.

(6) Bucardo, M., Saavedra, M. L. y Camarena, M. E. (2015). Hacia una comprensión de los conceptos de emprendedores y empresarios. Suma de Negocios, 6(13), 98-107.

(7) Cano, J. A. y Baena, J. J. (2015). Tendencias en el uso de las tecnologías de información y comunicación para la negociación internacional. Estudios Gerenciales, 31(136), 335-346. doi: 10.1016/j.estger.2015.03.003

(8) Contreras, O. E. y Rozo, I. (2015). Teletrabajo y sostenibilidad empresarial. Una reflexión desde la gerencia del talento humano en Colombia. Suma de Negocios, 6(13), 74-83. doi: 10.1016/j.sumneg.2015.08.006

(9) Fernández, S. (2013). It Takes Two to Tango: Commercial Relation Beyond Of Bilateral Agreement, China and Colombia to Sign a Free Trade Agreement. American Journal of Business and Management, 2(4), 275-295. doi: 10.11634/216796061302426

(10) Fernández, S. (2014a). Empirical result on firms' cluster integration: should firms evolve beyond their region?. International Journal of Trade, Economics and Finance, 5(3), 204-211. doi: 10.7763/IJTEF.2014.V5.372

(11) Fernández, S. (2014b). “产业集群一体化挑战研究.” CNKI JOURNAL, 1-141.

(12) Fernández, S. (2016). The Opportunities and Bottlenecks between China and Latin American Countries Economic and Trade Development. International Journal of Developing Societies, 5(2), 23-26. doi: 10.11634/216817831605783

(13) Fernández, S., Castillo, D. y Martínez, L. (2018). Clúster virtual: nueva alternativa a la competitividad eficaz en las empresas. Tendencias, 19(1), 164-186. doi: $10.22267 /$ rtend. 181901.92 
(14) Fernández, S., Diaz, L., Rodríguez, W. y Martinez, L. (2019). Influencia de la tecnología e información para el rendimiento de las Mipymes colombianas. Actualidad Contable Faces, 22(38), $25-45$.

(15) Fernández, S. y Martinez, L. (2018). Internationalization of colombian firms: competitive and productivity as a problem to enter the asian market. Asian Social Science, 14(1), 183-192. doi: 10.5539/ass.v14n1p183

(16) Flores, M. y Vanoni, G. (2016). Competencias Directivas requeridas por los CEO ante la complejidad de las organizaciones del siglo XXI. Suma de Negocios, 7(16), 113-124. doi: 10.1016/j.sumneg.2016.02.005

(17) García, P., Gálvez, E. y Maldonado, G. (2016). Efecto de la innovación en el crecimiento y el desempe no de las Mipymes de la Alianza del Pacífico. Un Estudio empírico. Estudios Gerenciales, 32(141), 326-335. doi: 10.1016/j.estger.2016.07.003

(18) Hernández. H., Martuscelli, J., Moctezuma, D., Muñoz, H. y Narro, J. (2015). Los desafíos de las universidades de América Latina y el Caribe ¿Qué somos y a dónde vamos? Perfiles educativos, 37(147), 202-218.

(19) Larrea, J. L. (2009). Las relaciones laborales ante el desafío de la innovacción: un nuevo compromiso. Boletín de estudios económicos, 64(196), 23-43.

(20) Martinez, L. A, y Fernández, S. (2018). Internal Communication Issues in the Firms: Does It Affect the Productivity? Review of European Studies, 10(2), 1-13. doi: 10.5539/res.v10n2p1

(21) Momberg, R. (2016). Análisis de los modelos de vinculación del código civil y la legislación de protección al consumidor. Hacia un principio general de protección de la parte débil en el derecho privado. Revista Chilena de Derecho, 43(2), 737-756. doi: 10.4067/S0718-34372016000200017

(22) Moreno, T. (2015). Las competencias del evaluador educativo. Revista de la Educacion Superior, 44(174), 101-126.

(23) Navarrete, J. E. (2017). Trump y la coyuntura económica global. Economía UNAM, 14(41, 4056.

(24) Ngono, R., Darcis, N., Muhammad, A. y Fernández, S. (2014). Port logistics in West and Central Africa: a strategic development under globalization. Open Journal of Applied Sciences, 4(2), 7684. doi: 10.4236/ojapps.2014.42009

(25) Pabón, J. A. (2016). Gestión del conocimiento y políticas de innovación. Revista La Propiedad Inmaterial, (22), 19-31. doi: 10.18601/16571959.n22.02

(26) Robayo, P. V. (2016). La innovación como proceso y su gestión en la organización: una aplicación para el sector gráfico colombiano. Suma de Negocios, 7(16), 125-140. doi: 10.1016/j.sumneg.2016.02.007

(27) San Martín, S., Jiménez, N. y Sánchez, E. (2016). La Evaluación del alumnado universitario en el Espacio Europeo de Educación Superior. Aula Abierta, 44(1), 7-14. doi: 10.1016/j.aula.2015.03.003 
(28) Santos, V. y García, T. (2016). Motivación del empresario y atención informativa en la internacionalización: un análisis regional en el sector español de piedra natural. European Research on Management and Business Economics, 22(1), 1-7. doi: 10.1016/j.iedee.2014.12.002

(29) Suárez, M. H. y Muñoz, H. (2016). ¿Qué pasa con los académicos? Revista de la Educacion Superior, 45(180), 1-22. doi: 10.1016/j.resu.2016.08.003

(30) Vanegas, J. G., Restrepo, J. A. y González, M. A. (2015). Negocios y comercio internacional: evidencias de investigación académica para Colombia. Suma de Negocios, 6(13), 84-91.

Cómo citar este artículo: Fernández, S., Martínez, L. y Ngono, R. (2020). El nivel educativo como eje fundamental para la internacionalización y desarrollo empresarial. Tendencias, 21(2), 106-123. https://doi.org/10.22267/rtend.202102.143 